\title{
Analysis of a Patch Model for the Dynamical Transmission of Echinococcosis
}

\author{
Kai Wang, ${ }^{1,2}$ Xueliang Zhang, ${ }^{2}$ Zhidong Teng, ${ }^{3}$ Lei Wang, ${ }^{2}$ and Liping Zhang ${ }^{2}$ \\ ${ }^{1}$ Department of Mathematics, North University of China, Taiyuan, Shanxi 030051, China \\ ${ }^{2}$ Department of Medical Engineering and Technology, Xinjiang Medical University, Urumqi 830011, China \\ ${ }^{3}$ College of Mathematics and System Sciences, Xinjiang University, Urumqi 830046, China \\ Correspondence should be addressed to Kai Wang; wangkaimath@sina.com
}

Received 18 January 2014; Accepted 4 February 2014; Published 18 March 2014

Academic Editor: Weiming Wang

Copyright (C) 2014 Kai Wang et al. This is an open access article distributed under the Creative Commons Attribution License, which permits unrestricted use, distribution, and reproduction in any medium, provided the original work is properly cited.

A patch model for echinococcosis due to dogs migration is proposed to explore the effect of dogs migration among patches on the spread of echinococcosis. We firstly define the basic reproduction number $R_{0}$. The mathematical results show that the dynamics of the model can be completely determined by $R_{0}$. If $R_{0}<1$, the disease-free equilibrium is globally asymptotically stable. When $R_{0}>1$, the model is permanence and endemic equilibrium is globally asymptotically stable. According to the simulations, it is shown that the larger diffusion of dogs from the lower epidemic areas to the higher prevalence areas can intensify the spread of echinococcosis. However, the larger diffusion of dogs from the higher prevalence areas to the lower epidemic areas can reduce the spread and is beneficial for disease control.

\section{Introduction}

Echinococcosis, which is often referred to as hydatid disease, is a parasitic disease that affects both humans and other mammals, such as sheep, dogs, rodents, and horses [1]. The two most clinically relevant species are Echinococcus granulosus and Echinococcus multilocularis, which cause cystic and alveolar echinococcosis respectively. Humans are incidental hosts and, in most cases, do not contribute to continuance of the parasite life cycle, except under unique circumstances [2].

The prevalent scope of echinococcosis in China is approximately 420 square kilometers, accounting for about $41.7 \%$ of the territory. The rate of incidence of echinococcosis has increased in the past decade. The operability of echinococcosis exceeds 10/100000 in each year. High-risk group subject to echinococcosis reaches up to 50 million, and the number of domestic animal amount being faced with the infection of echinococcosis is more than one hundred million, in which the amount of dogs is at least 5 million [3].

Mathematical modeling has become an important tool in analyzing the epidemiological characteristics of infectious disease and can provide useful control measures. Various models have been used to study different aspects of echinococcosis [4-16]. The models included varied primarily on the basis of six key features that were differentially incorporated in their design [17]. These are (1) the inclusion of a "latent" class (with time delay from host exposure to infectiousness); (2) an age structure for definitive and/or intermediate hosts; (3) the presence of density dependent constraints; (4) accounting for seasonality; (5) stochastic parameters; (6) inclusion of a spatial and risk structures.

In [18], in order to explore effective control and prevention measures authors proposed a deterministic model to study the transmission dynamics of echinococcosis in Xinjiang. The results showed that the dynamics of the model was completely determined by the basic reproductive number $R_{0}$. The model provided an approximate estimate of the basic reproduction number $R_{0}=1.67$.

Many epidemic models with population dispersal among patches have been proposed and studied (see [19-28]). Wang and Zhao [19] proposed an epidemic model to describe the dynamics of disease spread among patches due to population dispersal. The effect of population dispersal among $n$ patches on the spread of a disease was investigated by Jin and Wang in [20]. To understand the effect of transport-related infection on disease spread, an epidemic model for several 
regions which are connected by transportation of individuals has been proposed by Cui et al. in [21]. In [23], an SIS patch model with nonconstant transmission coefficients was formulated to investigate the effect of media coverage and human movement on the spread of infectious diseases among patches. Qiu [26] developed a mathematical model to explore the effect of host migration between two patches on the spread of a vector-host disease.

To date, few scholars have researched the echinococcosis transmission models with dogs migration among patches. Considering an increasing number of stray dogs, the dispersal is an essential trait for dogs population. Therefore, we expect to explore the effect of dogs migration among patches on the spread of echinococcosis.

The purpose of this paper is to model the transmission dynamics of echinococcosis spread between two patches due to dogs migration and describe the dynamics of the model. The remaining part of this paper is organized as follows. The model is presented in Section 2. The basic properties on the positivity and boundedness of solutions computing the basic reproduction number are in Section 3. In Section 4, we establish the global stability of the disease-free equilibrium for the model. In Section 5, we will apply the theory of permanence to obtain the permanence of the model. The global stability theorem of endemic equilibrium is stated and proved in Section 6. In Section 7, we give some examples to illustrate how the dogs migration affects the dynamics of echinococcosis. A brief discussion is given in Section 8 .

\section{Model Formulation}

In this section, we mainly formulate an epidemic model to describe the transmission dynamics of echinococcosis spread between two discrete patches due to dogs diffusion.

We firstly formulate a model for the spread of echinococcosis in the ith patch. It follows from [18] that the parameters of humans do not affect dynamical behaviors of echinococcosis model. Hence in the paper we only consider dogs, livestock, and Echinococcus eggs in our model. We divide the dogs population in the $i$ th patch into two classes: the susceptible population and the infected population denoted by $S_{D i}(t)$ and $I_{D i}(t)$, respectively. For livestock population, we divide the total livestock population in the $i$ th patch into two classes: susceptible and infectious denoted by $S_{L i}(t)$ and $I_{L i}(t)$, respectively. The density of Echinococcus eggs in the $i$ th patch is denoted by $x_{i}(t)$. Our assumptions on the dynamical transmission of echinococcosis in the $i$ th patch are demonstrated in the flowchart (Figure 1).

If there is no dogs migration among patches, that is, the patches are isolated, we suppose that the echinococcosis dynamics in $i$ th patch is governed by

$$
\begin{gathered}
\dot{S}_{D i}=A_{1 i}-\beta_{1 i} S_{D i} I_{L i}-d_{1 i} S_{D i}+\sigma_{i} I_{D i}, \\
\dot{I}_{D i}=\beta_{1 i} S_{D i} I_{L i}-\left(d_{1 i}+\sigma_{i}\right) I_{D i}, \\
\dot{S}_{L i}=A_{2 i}-\beta_{2 i} S_{L i} x_{i}-d_{2 i} S_{L i}, \\
\dot{I}_{L i}=\beta_{2 i} S_{L i} x_{i}-d_{2 i} I_{L i}, \\
\dot{x}_{i}=a_{i} I_{D i}-d_{i} x_{i} .
\end{gathered}
$$

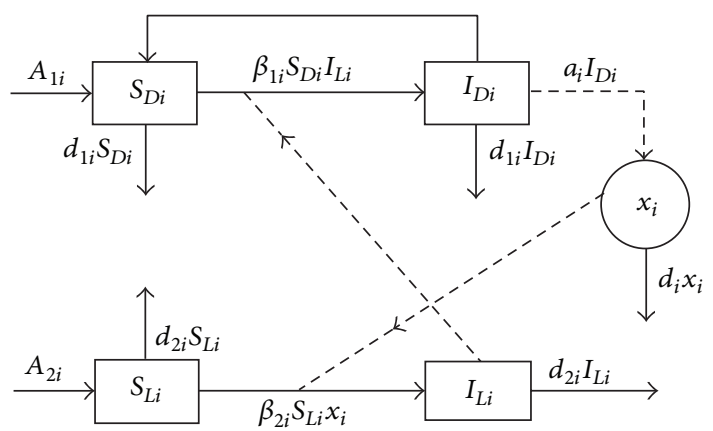

FIgURE 1: Transmission diagram for echinococcosis among dogs, livestock.

All parameters are assumed positive. For the dog population in the $i$ th patch, $A_{1 i}$ describes the annual recruitment rate; $d_{1 i}$ is the natural death rate; $\sigma_{i}$ denotes the recovery rate of transition from infected to noninfected dogs, including natural recovery rate and recovery due to anthelmintic treatment; $\beta_{1 i} S_{D i} I_{L i}$ describes the transmission of echinococcosis between susceptible dogs and infectious livestock after the ingestion of cyst-containing organs of the infected livestock. For the livestock population in the $i$ th patch, $A_{2 i}$ is the annual recruitment rate; $d_{2 i}$ is the death rate; $\beta_{2 i} S_{L i} x_{i}$ describes the transmission of echinococcosis to livestock by the ingestion of Echinococcus eggs in the environment. For Echinococcus eggs in the $i$ th patch, $a_{i}$ denotes released rate from infected dogs; $d_{i}$ is the mortality rate of eggs.

When two patches are connected, we assume that susceptible and infected dogs of every patch $i$ leave for patch $j$ at a per capita rate $D_{i}$. Then the dynamics of echinococcosis is governed by the following model:

$$
\begin{gathered}
\dot{S}_{D i}=A_{1 i}-\beta_{1 i} S_{D i} I_{L i}-d_{1 i} S_{D i}+\sigma_{i} I_{D i}-D_{i} S_{D i}+D_{j} S_{D j}, \\
\dot{I}_{D i}=\beta_{1 i} S_{D i} I_{L i}-\left(d_{1 i}+\sigma_{i}\right) I_{D i}-D_{i} I_{D i}+D_{j} I_{D j}, \\
\dot{S}_{L i}=A_{2 i}-\beta_{2 i} S_{L i} x_{i}-d_{2 i} S_{L i}, \quad i, j=1,2, \quad i \neq j . \\
\dot{I}_{L i}=\beta_{2 i} S_{L i} x_{i}-d_{2 i} I_{L i}, \\
\dot{x}_{i}=a_{i} I_{D i}-d_{i} x_{i} .
\end{gathered}
$$

Motivated by biological background of model (2), we always assume that all solutions of model (2) satisfy the following positive initial conditions:

$$
\begin{gathered}
S_{D i}(0)=S_{D i 0}>0, \quad I_{D i}(0)=I_{D i 0}>0, \\
S_{L i}(0)=S_{L i 0}>0, \quad I_{L i}(0)=I_{L i 0}>0, \\
x_{i}(0)=x_{i 0}>0 .
\end{gathered}
$$

We can easily prove that the solution of model (2) with initial conditions (3) satisfies $S_{D i}(t)>0, I_{D i}(t)>0, S_{L i}(t)>0$, and $I_{L i}(t)>0$ for all $t>0$. Here, we omit the proof. 


\section{Basic Properties and Basic Reproduction Number of the Model}

In this section, we mainly present the preliminary results and derive reproduction number for model (2). In order to investigate the dynamics of model (2), we begin with stating some results on model (1). Model (1) has been analyzed in [18]. Model (1) admits a disease-free equilibrium $E_{0 i}=$ $\left(S_{D i}^{0}, 0, S_{L i}^{0}, 0,0\right)$ and a unique positive equilibrium $E_{i}^{*}=$ $\left(S_{D i}^{*}, I_{D i}^{*}, S_{L i}^{*}, I_{L i}^{*}, x_{i}^{*}\right)$, where

$$
\begin{gathered}
S_{D i}^{0}=\frac{A_{1 i}}{d_{1 i}}, \quad S_{L i}^{0}=\frac{A_{2 i}}{d_{2 i}}, \\
S_{D i}^{*}=\frac{d_{2 i}\left(d_{1 i}+\sigma_{i}\right)\left(A_{1 i} \beta_{2 i} a_{i}+d_{1 i} d_{2 i} d_{i}\right)}{a_{i} \beta_{2 i} d_{1 i}\left(\beta_{1 i} A_{2 i}+d_{1 i} d_{2 i}+d_{2 i} \sigma_{i}\right)}, \\
I_{D i}^{*}=\frac{a_{i} \beta_{1 i} \beta_{2 i} A_{1 i} A_{2 i}-\left(d_{1 i}+\sigma_{i}\right) d_{1 i} d_{2 i}^{2} d_{i}}{a_{i} d_{1 i} \beta_{2 i}\left(\beta_{1 i} A_{2 i}+d_{1 i} d_{2 i}+d_{2 i} \sigma_{i}\right)}, \\
S_{L i}^{*}=\frac{d_{i} d_{1 i}\left(\beta_{1 i} A_{2 i}+d_{1 i} d_{2 i}+d_{2 i} \sigma_{i}\right)}{\beta_{1 i}\left(a_{i} A_{1 i} \beta_{2 i}+d_{i} d_{1 i} d_{2 i}\right)}, \\
I_{L i}^{*}=\frac{a_{i} \beta_{1 i} \beta_{2 i} A_{1 i} A_{2 i}-\left(d_{1 i}+\sigma_{i}\right) d_{1 i} d_{2 i}^{2} d_{i}}{d_{2 i} \beta_{1 i}\left(A_{1 i} \beta_{2 i} a_{i}+d_{1 i} d_{2 i} d_{i}\right)}, \\
x_{i}^{*}=\frac{a_{i} \beta_{1 i} \beta_{2 i} A_{1 i} A_{2 i}-\left(d_{1 i}+\sigma_{i}\right) d_{1 i} d_{2 i}^{2} d_{i}}{d_{1 i} \beta_{2 i} d\left(\beta_{1 i} A_{2 i}+d_{1 i} d_{2 i}+d_{2 i} \sigma_{i}\right)} .
\end{gathered}
$$

The reproduction number of model (1) is established in [18], which can be expressed as

$$
R_{0 i}=\sqrt[3]{\frac{\beta_{1 i} \beta_{2 i} A_{1 i} A_{2 i} a_{i}}{\left(d_{1 i}+\sigma_{i}\right) d_{1 i} d_{2 i}^{2} d_{i}}} .
$$

From Theorems 3 and 5 in [18], we can obtain the following lemma.

Lemma 1. Considering model (1), one has that

(a) if $R_{0 i}<1$, then disease-free equilibrium $E_{0 i}$ is globally asymptotically stable;

(b) if $R_{0 i}>1$, then positive equilibrium $E_{i}^{*}$ is globally asymptotically stable.

In order to obtain our main results, we need the following lemma. Consider the following linear equation:

$$
\begin{aligned}
& \widetilde{N}_{D 1}^{\prime}(t)=A_{11}-d_{11} \widetilde{N}_{D 1}(t)-D_{1} \widetilde{N}_{D 1}(t)+D_{2} \widetilde{N}_{D 2}(t), \\
& \widetilde{N}_{D 2}^{\prime}(t)=A_{12}-d_{12} \widetilde{N}_{D 2}(t)-D_{2} \widetilde{N}_{D 2}(t)+D_{1} \widetilde{N}_{D 1}(t) .
\end{aligned}
$$

We have the following result on system (6).

Lemma 2. System (6) has a unique equilibrium $N_{D}^{0}\left(N_{D 1}^{0}, N_{D 2}^{0}\right)$ which is globally stable, where

$$
\begin{aligned}
& N_{D 1}^{0}=\frac{A_{11}\left(d_{12}+D_{2}\right)+A_{12} D_{2}}{d_{11} d_{12}+d_{11} D_{2}+d_{12} D_{1}}, \\
& N_{D 2}^{0}=\frac{A_{12}\left(d_{11}+D_{1}\right)+A_{11} D_{1}}{d_{11} d_{12}+d_{11} D_{2}+d_{12} D_{1}} .
\end{aligned}
$$

Proof. The Jacobian matrix of $(6)$ at $\left(N_{D 1}^{0}, N_{D 2}^{0}\right)$ is

$$
J\left(N_{D}^{0}\right)=\left(\begin{array}{cc}
-\left(d_{11}+D_{1}\right) & D_{2} \\
D_{1} & -\left(d_{12}+D_{2}\right)
\end{array}\right) .
$$

By simple calculations, the corresponding characteristic equation is

$$
\Phi(\lambda)=\lambda^{2}+a_{1} \lambda+a_{0}=0
$$

where

$$
\begin{gathered}
a_{1}=d_{11}+d_{12}+D_{1}+D_{2}>0, \\
a_{0}=d_{11} d_{12}+d_{11} D_{2}+d_{12} D_{1}>0 .
\end{gathered}
$$

Therefore, all roots of $\Phi(\lambda)$ have negative real parts, and hence $N^{0}\left(N_{D 1}^{0}, N_{D 2}^{0}\right)$ is globally stable.

For any $\varepsilon>0$, we define region $\Gamma_{\varepsilon}$ as follows:

$$
\begin{gathered}
\Gamma_{\varepsilon}=\left\{\left(S_{D 1}, I_{D 1}, S_{L 1}, I_{L 1}, x_{1}, S_{D 2}, I_{D 2}, S_{L 2}, I_{L 2}, x_{2}\right) \in \mathbb{R}_{+}^{10},\right. \\
S_{D i}+I_{D i} \leq N_{D i}^{0}+\varepsilon, S_{L i}+I_{L i} \leq S_{L i}^{0}+\varepsilon, \\
\left.x_{i} \leq \frac{a_{i}}{d_{i}} N_{D i}^{0}+\left(1+\frac{a_{i}}{d_{i}}\right) \varepsilon, i=1,2\right\} .
\end{gathered}
$$

On the ultimate boundedness of solutions for model (2), we have the following result.

Lemma 3. All solutions of model (2) with initial condition (3) ultimately turn into region $\Gamma_{\varepsilon}$ as $t \rightarrow \infty$.

Proof. Let $\quad\left(S_{D 1}(t), I_{D 1}(t), S_{L 1}(t), I_{L 1}(t), x_{1}(t), S_{D 2}(t), I_{D 2}(t)\right.$, $\left.S_{L 2}(t), I_{L 2}(t), x_{2}(t)\right)$ be any solution of model (2) with initial conditions (3) and let $N_{D i}(t)=S_{D i}(t)+I_{D i}(t), i=1,2$. From model (2) we have

$$
\begin{aligned}
& \dot{N}_{D 1}(t)=A_{11}-d_{11} N_{D 1}(t)-D_{1} N_{D 1}(t)+D_{2} N_{D 2}(t), \\
& \dot{N}_{D 2}(t)=A_{12}-d_{12} N_{D 2}(t)-D_{2} N_{D 2}(t)+D_{1} N_{D 1}(t),
\end{aligned}
$$

and then from Lemma 2 we have $\lim _{t \rightarrow \infty} N_{D i}(t)=N_{D i}^{0}, i=$ 1,2 . Hence, for any $\varepsilon>0$, there is a $t_{1}>0$ such that

$$
S_{D i}(t)+I_{D i}(t) \leq N_{D i}^{0}+\varepsilon, \quad i=1,2, \forall t \geq t_{1} .
$$

From the third and fourth equations of model (2), we have

$$
\frac{d\left(S_{L i}(t)+I_{L i}(t)\right)}{d t}=A_{2 i}-d_{2 i}\left(S_{L i}(t)+I_{L i}(t)\right),
$$

and therefore, there exists a $t_{2}>0$ such that

$$
S_{L i}(t)+I_{L i}(t) \leq S_{L i}^{0}+\varepsilon, \quad i=1,2, \forall t \geq t_{2} .
$$

Finally, from the fifth equation of model (2), we have

$$
\dot{x}_{i}(t) \leq a_{i}\left(N_{D i}^{0}+\varepsilon\right)-d_{i} x_{i}(t), \quad i=1,2, \forall t \geq t_{1},
$$


and then there is a $t_{3}>t_{1}$ such that

$$
\begin{aligned}
x_{i}(t) & \leq \frac{a_{i}}{d_{i}}\left(N_{D i}^{0}+\varepsilon\right)+\varepsilon \\
& =\frac{a_{i}}{d_{i}} N_{D i}^{0}+\left(1+\frac{a_{i}}{d_{i}}\right) \varepsilon, \quad i=1,2, \forall t \geq t_{3} .
\end{aligned}
$$

Let $t^{*}=\max \left\{t_{2}, t_{3}\right\}$, and then for all $t>t^{*}$ we have

$$
\begin{gathered}
\left(\left(S_{D 1}(t), I_{D 1}(t), S_{L 1}(t), I_{L 1}(t), x_{1}(t), S_{D 2}(t),\right.\right. \\
\left.\left.I_{D 2}(t), S_{L 2}(t), I_{L 2}(t), x_{2}(t)\right)\right) \in \Gamma_{\varepsilon} .
\end{gathered}
$$

This completes the proof of Lemma 3.

According to Lemma 3, all feasible solutions of model (2) enter or remain in the region $\Gamma_{\varepsilon}$ as $t$ becomes large enough. In what follows, the dynamics of model (2) can be considered only in $\Gamma_{\varepsilon}$.

Simple algebraic calculation shows that model (2) always has a unique disease-free equilibrium $E_{0}\left(N_{D 1}^{0}, 0, S_{L 1}^{0}, 0,0\right.$, $\left.N_{D 2}^{0}, 0, S_{L 2}^{0}, 0,0\right)$. According to the concepts of next generation matrix and reproduction number presented in $[29,30]$, we define

$$
\mathscr{F}=\left(\begin{array}{c}
\beta_{11} S_{D 1} I_{L 1} \\
\beta_{21} S_{L 1} x_{1} \\
a_{1} I_{D 1} \\
\beta_{12} S_{D 2} I_{L 2} \\
\beta_{22} S_{L 2} x_{2} \\
a_{2} I_{D 2}
\end{array}\right),
$$

$$
\begin{aligned}
& M_{11}=\left(\begin{array}{ccc}
0 & \frac{\Delta\left(A_{11} d_{12}+A_{11} D_{2}+A_{12} D_{2}\right)}{d_{21}\left(d_{11} d_{12}+d_{11} D_{2}+d_{12} D_{1}\right)} & 0 \\
0 & 0 & \frac{\Delta \beta_{21} A_{21}}{d_{21} d_{1}} \\
d_{1} d_{2} d_{21} d_{22} a_{1}\left(d_{12}+\sigma_{2}+D_{2}\right) & 0 & 0
\end{array}\right), \\
& M_{12}=\left(\begin{array}{ccc}
0 & 0 & 0 \\
0 & 0 & 0 \\
d_{1} d_{2} d_{21} d_{22} a_{1} D_{2} & 0 & 0
\end{array}\right), \quad M_{21}=\left(\begin{array}{ccc}
0 & 0 & 0 \\
0 & 0 & 0 \\
d_{1} d_{2} d_{21} d_{22} a_{2} D_{1} & 0 & 0
\end{array}\right) \text {, } \\
& M_{22}=\left(\begin{array}{ccc}
0 & \frac{\Delta \beta_{12}\left(A_{11} D_{1}+A_{12} d_{11}+A_{12} D_{1}\right)}{d_{22}\left(d_{11} d_{12}+d_{11} D_{2}+d_{12} D_{1}\right)} & 0 \\
0 & 0 & \frac{\Delta \beta_{22} A_{22}}{d_{22} d_{2}} \\
d_{1} d_{2} d_{21} d_{22} a_{2}\left(d_{11}+\sigma_{1}+D_{1}\right) & 0 & 0
\end{array}\right) .
\end{aligned}
$$

Denote $\Delta=d_{1} d_{2} d_{21} d_{22}\left[d_{11}\left(d_{12}+\sigma_{2}+D_{2}\right)+\sigma_{1}\left(d_{12}+\sigma_{2}+\right.\right.$ $\left.\left.D_{2}\right)+D_{1}\left(d_{12}+\sigma_{2}\right)\right]$. After extensive algebraic calculations, we can obtain

$$
F V^{-1}=\frac{1}{\Delta}\left(\begin{array}{ll}
M_{11} & M_{12} \\
M_{21} & M_{22}
\end{array}\right)
$$

where 
From the proof of Theorem 2 in [30], it follows that

$$
R_{0}<1\left(R_{0}=1, R_{0}>1\right) \Longleftrightarrow s(J)<1(s(J)=0, s(J)>0),
$$

$$
\begin{aligned}
J & =F-V \\
& =\left(\begin{array}{ccc}
-\left(d_{11}+\sigma_{1}+D_{1}\right) & \beta_{11} N_{D 1}^{0} & 0 \\
0 & -d_{21} & \beta_{21} S_{L 1}^{0} \\
a_{1} & 0 & -d_{1} \\
D_{1} & 0 & 0 \\
0 & 0 & 0 \\
0 & 0 & 0
\end{array}\right.
\end{aligned}
$$

and $s(J)$ is the maximum real part of the eigenvalues of matrix J.

Using Theorem 2 in [30], we can easily obtain the following stability result.

Theorem 4. For model (2), one has that

(a) if $R_{0}<1$, then disease-free equilibrium $E_{0}$ is locally asymptotically stable;

(b) if $R_{0}>1$, then disease-free equilibrium $E_{0}$ is unstable.

\section{Global Stability of the \\ Disease-Free Equilibrium}

We start by considering the global stability of disease-free equilibrium $E_{0}$ when $R_{0}<1$.

Theorem 5. The disease-free equilibrium $E_{0}$ of model (2) is globally asymptotically stable in $\Gamma_{\varepsilon}$ if $R_{0}<1$.

Proof. From Theorem 4 we find that disease-free equilibrium $E_{0}$ is locally asymptotically stable if $R_{0}<1$. In the following we only need to prove the global attractiveness of $E_{0}$. From (24) we can see that if $R_{0}<1$, then $s(J)<0$. Hence, there is a small enough number $\varepsilon>0$ such that $s\left(J_{\varepsilon}\right)<0$, where $J_{\varepsilon}=J+\varepsilon J_{1}$ and

$$
J_{1}=\left(\begin{array}{cccccc}
0 & \beta_{11} & 0 & 0 & 0 & 0 \\
0 & 0 & \beta_{21} & 0 & 0 & 0 \\
0 & 0 & 0 & 0 & 0 & 0 \\
0 & 0 & 0 & 0 & \beta_{12} & 0 \\
0 & 0 & 0 & 0 & 0 & \beta_{22} \\
0 & 0 & 0 & 0 & 0 & 0
\end{array}\right)
$$

Let $\left(S_{D 1}(t), I_{D 1}(t), S_{L 1}(t), I_{L 1}(t), x_{1}(t), S_{D 2}(t), I_{D 2}(t), S_{L 2}(t)\right.$, $\left.I_{L 2}(t), x_{2}(t)\right)$ be any solution of model (2) in $\Gamma_{\varepsilon}$, then

$$
S_{D i}(t) \leq N_{D i}^{0}+\varepsilon, \quad S_{L i}(t) \leq S_{L i}^{0}+\varepsilon, \quad i=1,2, \forall t \geq 0 .
$$

where

$$
\left.\begin{array}{ccc}
D_{2} & 0 & 0 \\
0 & 0 & 0 \\
0 & 0 & 0 \\
-\left(d_{12}+\sigma_{2}+D_{2}\right) & \beta_{21} N_{D 2}^{0} & 0 \\
0 & -d_{22} & \beta_{22} S_{L 2}^{0} \\
a_{2} & 0 & -d_{2}
\end{array}\right)
$$

From model (2), it follows that

$$
\begin{gathered}
\dot{I}_{D 1} \leq \beta_{11}\left(N_{D 1}^{0}+\varepsilon\right) I_{L 1}-\left(d_{11}+\sigma_{1}\right) I_{D 1}-D_{1} I_{D 1}+D_{2} I_{D 2}, \\
\dot{I}_{L 1} \leq \beta_{21}\left(S_{L 1}^{0}+\varepsilon\right) x_{1}-d_{21} I_{L 1}, \\
\dot{x}_{1} \leq a_{1} I_{D 1}-d_{1} x_{1}, \\
\dot{I}_{D 2} \leq \beta_{12}\left(N_{D 2}^{0}+\varepsilon\right) I_{L 2}-\left(d_{12}+\sigma_{2}\right) I_{D 2}-D_{2} I_{D 2}+D_{1} I_{D 1}, \\
\dot{I}_{L 2} \leq \beta_{22}\left(S_{L 2}^{0}+\varepsilon\right) x_{2}-d_{22} I_{L 2}, \\
\dot{x}_{2} \leq a_{2} I_{D 2}-d_{2} x_{2} .
\end{gathered}
$$

Define an auxiliary linear system:

$$
\begin{gathered}
\bar{I}_{D 1}^{\prime}=\beta_{11}\left(N_{D 1}^{0}+\varepsilon\right) \bar{I}_{L 1}-\left(d_{11}+\sigma_{1}\right) \bar{I}_{D 1}-D_{1} \bar{I}_{D 1}+D_{2} \bar{I}_{D 2}, \\
\bar{I}_{L 1}^{\prime}=\beta_{21}\left(S_{L 1}^{0}+\varepsilon\right) \bar{x}_{1}-d_{21} \bar{I}_{L 1}, \\
\bar{x}_{1}^{\prime}=a_{1} \bar{I}_{D 1}-d_{1} \bar{x}_{1}, \\
\bar{I}_{D 2}^{\prime}=\beta_{12}\left(N_{D 2}^{0}+\varepsilon\right) \bar{I}_{L 2}-\left(d_{12}+\sigma_{2}\right) \bar{I}_{D 2}-D_{2} \bar{I}_{D 2}+D_{1} \bar{I}_{D 1}, \\
\bar{I}_{L 2}^{\prime}=\beta_{22}\left(S_{L 2}^{0}+\varepsilon\right) \bar{x}_{2}-d_{22} \bar{I}_{L 2}, \\
\bar{x}_{2}^{\prime}=a_{2} \bar{I}_{D 2}-d_{2} \bar{x}_{2} .
\end{gathered}
$$

Since system (29) is a linear system, the globally stability of origin is determined by the stability of matrix $J_{\varepsilon}$. Since $s\left(J_{\varepsilon}\right)<$ 0 , then all the eigenvalues of matrix $J_{\varepsilon}$ have negative real parts. It then follows that each solution of (29) satisfies

$$
\begin{gathered}
\lim _{t \rightarrow+\infty} \bar{I}_{D i}(t)=0, \quad \lim _{t \rightarrow+\infty} \bar{I}_{L i}(t)=0, \\
\lim _{t \rightarrow+\infty} \bar{x}_{i}(t)=0, \quad i=1,2 .
\end{gathered}
$$

By the comparison principle we have

$$
\begin{gathered}
\lim _{t \rightarrow+\infty} I_{D i}(t)=0, \quad \lim _{t \rightarrow+\infty} I_{L i}(t)=0, \\
\lim _{t \rightarrow+\infty} x_{i}(t)=0, \quad i=1,2 .
\end{gathered}
$$


Then the limiting system of model (2) is

$$
\begin{gathered}
\dot{S}_{D 1}=A_{11}-d_{11} S_{D 1}-D_{1} S_{D 1}+D_{2} S_{D 2} \\
\dot{S}_{D 2}=A_{12}-d_{12} S_{D 2}-D_{2} S_{D 2}+D_{1} S_{D 1} \\
\dot{S}_{L 1}=A_{21}-d_{21} S_{L 1} \\
\dot{S}_{L 2}=A_{22}-d_{22} S_{L 2} .
\end{gathered}
$$

By Lemma 2 we find that there is a unique equilibrium $\left(N_{D 1}^{0}, N_{D 2}^{0}, S_{L 1}^{0}, S_{L 2}^{0}\right)$ of system (32), which is globally asymptotically stable. Thus, according to the theory of asymptotic autonomous systems [31], we finally obtain that disease-free equilibrium $E_{0}$ is globally asymptotically stable for model (2) when $R_{0}<1$. This completes the proof of Theorem 5 .

\section{Permanence}

We now turn to the case where $R_{0}>1$. We first establish the permanence for model (2).

Theorem 6. Let $D_{i}>0, i=1,2$. If $R_{0}>1$, then model (2) is permanent. Furthermore, model (2) also has at least one positive equilibrium $E^{*}\left(S_{D 1}^{*}, I_{D 1}^{*}, S_{L 1}^{*}, I_{L 1}^{*}, x_{1}^{*}, S_{D 2}^{*}, I_{D 2}^{*}, S_{L 2}^{*}, I_{L 2}^{*}, x_{2}^{*}\right)$.

Proof. Define

$$
\begin{gathered}
X=\left\{\left(S_{D 1}, I_{D 1}, S_{L 1}, I_{L 1}, x_{1}, S_{D 2}, I_{D 2}, S_{L 2}, I_{L 2}, x_{2}\right):\right. \\
\left.S_{D i} \geq 0, I_{D i} \geq 0, S_{L i} \geq 0, I_{L i} \geq 0, x_{i} \geq 0, i=1,2\right\}, \\
X_{0}=\left\{\left(S_{D 1}, I_{D 1}, S_{L 1}, I_{L 1}, x_{1}, S_{D 2}, I_{D 2}, S_{L 2}, I_{L 2}, x_{2}\right):\right. \\
\left.S_{D i}>0, I_{D i}>0, S_{L i}>0, I_{L i}>0, x_{i}>0, i=1,2\right\}, \\
\partial X_{0}=X \backslash X_{0}, \\
M_{\partial}=\left\{\left(S_{D 1}(0), I_{D 1}(0), S_{L 1}(0), I_{L 1}(0), x_{1}(0),\right.\right. \\
\left.S_{D 2}(0), I_{D 2}(0), S_{L 2}(0), I_{L 2}(0), x_{2}(0)\right): \\
\left(S_{D 1}(t), I_{D 1}(t), S_{L 1}(t), I_{L 1}(t), x_{1}(t),\right. \\
\left.S_{D 2}(t), I_{D 2}(t), S_{L 2}(t), I_{L 2}(t), x_{2}(t)\right)
\end{gathered}
$$

satisfies model (2),

$$
\begin{gathered}
\left(S_{D 1}(t), I_{D 1}(t), S_{L 1}(t), I_{L 1}(t), x_{1}(t), S_{D 2}(t),\right. \\
\left.\left.I_{D 2}(t), S_{L 2}(t), I_{L 2}(t), x_{2}(t)\right) \in \partial X_{0}, \forall t \geq 0\right\} .
\end{gathered}
$$

In order to prove Theorem 6 , it suffices to show that $\partial X_{0}$ repels uniformly the solutions of $X_{0}$.

Firstly, by the form of model (2), it is easy to see that both $X$ and $X_{0}$ are positively invariant. Clearly, $\partial X_{0}$ is relatively closed in $X$. Furthermore, model (2) is point dissipative (see Lemma 3).

We now show that if $D_{i}>0, i=1,2$, then

$$
\begin{gathered}
M_{\partial}=\left\{\left(S_{D 1}, 0, S_{L 1}, 0,0, S_{D 2}, 0, S_{L 2}, 0,0\right):\right. \\
\left.S_{D i} \geq 0, S_{L i} \geq 0, i=1,2\right\} .
\end{gathered}
$$

Assume

$$
\begin{aligned}
& \left(S_{D 1}(0), I_{D 1}(0), S_{L 1}(0), I_{L 1}(0), x_{1}(0),\right. \\
& \left.\quad S_{D 2}(0), I_{D 2}(0), S_{L 2}(0), I_{L 2}(0), x_{2}(0)\right) \in M_{\partial} .
\end{aligned}
$$

It suffices to show that

$$
\begin{aligned}
I_{D 1}(t) & =I_{L 1}(t)=x_{1}(t)=I_{D 2}(t) \\
& =I_{L 2}(t)=x_{2}(t)=0, \quad \forall t \geq 0 .
\end{aligned}
$$

Suppose not, then there exists a $t_{0} \geq 0$ such that at least one of $I_{D 1}\left(t_{0}\right), I_{L 1}\left(t_{0}\right), x_{1}\left(t_{0}\right), I_{D 2}\left(t_{0}\right), I_{L 2}\left(t_{0}\right)$, or $x_{2}\left(t_{0}\right)$ is greater than zero. Here we only consider the case $I_{D 1}\left(t_{0}\right)>0, I_{D 2}\left(t_{0}\right)=0$, $S_{D i}\left(t_{0}\right)=0, S_{L i}\left(t_{0}\right)=0, I_{L i}\left(t_{0}\right)=0$, and $x_{i}\left(t_{0}\right)=0, i=1,2$. The other case can be deduced in the same way. Since

$$
\begin{gathered}
\dot{S}_{D i}\left(t_{0}\right)=A_{1 i}-\beta_{1 i} S_{D i}\left(t_{0}\right) I_{L i}\left(t_{0}\right)-d_{1 i} S_{D i}\left(t_{0}\right)+\sigma_{i} S_{D i}\left(t_{0}\right) \\
+D_{i} S_{D i}\left(t_{0}\right)-D_{j} S_{D j}\left(t_{0}\right) \geq A_{1 i}>0, \\
\dot{S}_{L i}\left(t_{0}\right)=A_{2 i}-\beta_{2 i} S_{L i}\left(t_{0}\right) x_{i}\left(t_{0}\right)-d_{2 i} S_{L i}\left(t_{0}\right)=A_{2 i}>0, \\
\dot{x}_{1}\left(t_{0}\right)=a I_{D 1}\left(t_{0}\right)-d_{1} x_{1}\left(t_{0}\right)=a I_{D 1}\left(t_{0}\right)>0, \\
\dot{I}_{D 1}(t) \geq-\left(d_{11}+\sigma_{1}+D_{1}\right) I_{D 1}(t), \quad i=1,2, i \neq j,
\end{gathered}
$$

it follows that there is an $\epsilon_{0}>0$ small enough such that $S_{D i}(t)>0, S_{L i}(t)>0, x_{1}(t)>0$, and $I_{D 1}(t)>0, i=1,2$, for all $t_{0}<t<t_{0}+\epsilon_{0}$. If $I_{L 1}\left(t_{0}+\left(\epsilon_{0} / 2\right)\right)>0$, then we have

$$
\dot{I}_{L 1}(t) \geq-d_{21} I_{L 1}(t) \text {. }
$$

This means that $I_{L 1}(t)>0$ for all $t \geq t_{0}+\left(\epsilon_{0} / 2\right)$. If $I_{L 1}\left(t_{0}+\right.$ $\left.\left(\epsilon_{0} / 2\right)\right)=0$, it then follows from model (2) that

$$
\dot{I}_{L 1}\left(t_{0}+\frac{\epsilon_{0}}{2}\right)=\beta_{21} S_{L 1}\left(t_{0}+\frac{\epsilon_{0}}{2}\right) x_{1}\left(t_{0}+\frac{\epsilon_{0}}{2}\right)>0 .
$$

It then follows that there exists an $\epsilon_{1}<\left(\epsilon_{0} / 2\right)$ such that

$$
I_{L 1}(t)>0, \quad \forall t_{0}+\frac{\epsilon_{0}}{2}<t<t_{0}+\frac{\epsilon_{0}}{2}+\epsilon_{1} .
$$

By the same way we can obtain that there exists an $\epsilon_{2}<\epsilon_{1}$ such that

$$
I_{D 2}(t)>0, \quad \forall t_{0}+\frac{\epsilon_{0}}{2}<t<t_{0}+\frac{\epsilon_{0}}{2}+\epsilon_{2} .
$$

If $x_{2}\left(t_{0}+\left(\epsilon_{0} / 2\right)+\left(\epsilon_{2} / 2\right)\right)>0$, then we have

$$
\dot{x}_{2}(t) \geq d_{2} x_{2}(t) .
$$

This means that $x_{2}(t)>0$ for all $t>t_{0}+\left(\epsilon_{0} / 2\right)+\left(\epsilon_{2} / 2\right)$; if $x_{2}\left(t_{0}+\left(\epsilon_{0} / 2\right)+\left(\epsilon_{2} / 2\right)\right)=0$, it then follows from model (2) that

$$
\dot{x}_{2}\left(t_{0}+\frac{\epsilon_{0}}{2}+\frac{\epsilon_{2}}{2}\right)=a_{2} I_{D 2}\left(t_{0}+\frac{\epsilon_{0}}{2}+\frac{\epsilon_{2}}{2}\right)>0 .
$$

It then follows that there exists an $\epsilon_{3}<\left(\epsilon_{2} / 2\right)$ such that

$$
x_{2}(t)>0, \quad \forall t_{0}+\frac{\epsilon_{0}}{2}+\frac{\epsilon_{2}}{2}<t<t_{0}+\frac{\epsilon_{0}}{2}+\frac{\epsilon_{2}}{2}+\epsilon_{3} .
$$


By the same way we can obtain that there exists an $\epsilon_{4}<\left(\epsilon_{3} / 2\right)$ such that

$$
\begin{gathered}
I_{L 2}(t)>0, \\
\forall t_{0}+\frac{\epsilon_{0}}{2}+\frac{\epsilon_{2}}{2}+\frac{\epsilon_{3}}{2}<t<t_{0}+\frac{\epsilon_{0}}{2}+\frac{\epsilon_{2}}{2}+\frac{\epsilon_{3}}{2}+\epsilon_{4} .
\end{gathered}
$$

Thus for all $t \in\left(t_{0}+\left(\epsilon_{0} / 2\right)+\left(\epsilon_{2} / 2\right)+\left(\epsilon_{3} / 2\right), t_{0}+\left(\epsilon_{0} / 2\right)\right.$ $\left.+\left(\epsilon_{2} / 2\right)+\left(\epsilon_{3} / 2\right)+\epsilon_{4}\right)$ we have $S_{D i}(t)>0, I_{D i}(t)>0$,
$S_{L i}(t)>0, I_{L i}(t)>0$, and $x_{i}(t)>0, i=1,2$. This contradicts the assumption that $\left(S_{D 1}(0), I_{D 1}(0), S_{L 1}(0), I_{L 1}(0)\right.$, $\left.x_{1}(0), S_{D 2}(0), I_{D 2}(0), S_{L 2}(0), I_{L 2}(0), x_{2}(0)\right) \in M_{\partial}$. This proves (35).

From (24) we can see that if $R_{0}>1$, then $s(J)>0$. Hence, there is a small enough number $\theta>0$ such that $s\left(J_{\theta}\right)>0$, where $J_{\theta}=J-\theta J_{1}$ and $J_{1}$ is given by (26). Let

$$
\begin{aligned}
g(x) & =\left(\begin{array}{l}
g_{1}(x) \\
g_{2}(x) \\
g_{3}(x) \\
g_{4}(x)
\end{array}\right) \\
& =\left(\begin{array}{c}
\frac{A_{11}\left(d_{12}+D_{2}+\beta_{12} x\right)+A_{12} D_{2}}{\left(\beta_{11} x+d_{11}\right)\left(\beta_{12} x+d_{12}\right)+D_{1}\left(\beta_{12} x+d_{12}\right)+D_{2}\left(\beta_{11}+d_{11}\right)} \\
\frac{A_{12}\left(d_{11}+D_{1}+\beta_{11} x\right)+A_{11} D_{1}}{\left(\beta_{11} x+d_{11}\right)\left(\beta_{12} x+d_{12}\right)+D_{1}\left(\beta_{12} x+d_{12}\right)+D_{2}\left(\beta_{11}+d_{11}\right)} \\
\frac{A_{12}}{d_{21}+\beta_{21} x} \\
\frac{A_{22}}{d_{22}+\beta_{22} x}
\end{array}\right),
\end{aligned}
$$

and we can see the fact that $\lim _{x \rightarrow 0} g(x)=\left(N_{D 1}^{0}, N_{D 2}^{0}\right.$, $\left.S_{L 1}^{0}, S_{L 2}^{0}\right)^{T}$. Hence we can choose $\delta>0$ small enough such that

$$
\begin{gathered}
g_{1}(\delta)=\left(A_{11}\left(d_{12}+D_{2}+\beta_{12} \delta\right)+A_{12} D_{2}\right) \\
\times\left(\left(\beta_{11} \delta+d_{11}\right)\left(\beta_{12} \delta+d_{12}\right)+D_{1}\left(\beta_{12} \delta+d_{12}\right)\right. \\
\left.+D_{2}\left(\beta_{11}+d_{11}\right)\right)^{-1}>N_{D 1}^{0}-\theta, \\
g_{2}(\delta)=\left(A_{12}\left(d_{11}+D_{1}+\beta_{11} \delta\right)+A_{11} D_{1}\right) \\
\times\left(\left(\beta_{11} \delta+d_{11}\right)\left(\beta_{12} \delta+d_{12}\right)+D_{1}\left(\beta_{12} \delta+d_{12}\right)\right. \\
\left.+D_{2}\left(\beta_{11}+d_{11}\right)\right)^{-1}>N_{D 2}^{0}-\theta, \\
g_{3}(\delta)=\frac{A_{12}}{d_{21}+\beta_{21} \delta}>S_{L 1}^{0}-\theta, \\
g_{4}(\delta)=\frac{A_{22}}{d_{22}+\beta_{22} \delta}>S_{L 2}^{0}-\theta .
\end{gathered}
$$

Suppose $\left(S_{D 1}(t), I_{D 1}(t), S_{L 1}(t), I_{L 1}(t), x_{1}(t), S_{D 2}(t), I_{D 2}(t), S_{L 2}(t)\right.$, $\left.I_{L 2}(t), x_{2}(t)\right)$ is a solution of model (2) with $\left(S_{D 1}(0), I_{D 1}(0)\right.$, $\left.S_{L 1}(0), I_{L 1}(0), x_{1}(0), S_{D 2}(0), I_{D 2}(0), S_{L 2}(0), I_{L 2}(0), x_{2}(0)\right) \in X_{0}$. We now claim that

$$
\begin{gathered}
\underset{t \rightarrow \infty}{\limsup \max }\left\{I_{D 1}(t), I_{L 1}(t), x_{1}(t), I_{D 2}(t),\right. \\
\left.I_{L 2}(t), x_{2}(t)\right\}>\delta .
\end{gathered}
$$

Suppose, for the sake of contradiction, that there exists a $T>$ 0 such that $I_{D i} \leq \delta, I_{L i} \leq \delta$, and $x_{i}(t) \leq \delta, i=1,2$, for all $t \geq T$. Then by model (2) we have

$$
\begin{aligned}
\dot{S}_{D 1}(t) \geq & A_{11}-\left(\beta_{11} \delta+d_{11}\right) S_{D 1}-D_{1} S_{D 1}+D_{2} S_{D 2} \\
\dot{S}_{D 2}(t) \geq & A_{12}-\left(\beta_{12} \delta+d_{12}\right) S_{D 2}-D_{2} S_{D 2}+D_{1} S_{D 1}, \\
& \dot{S}_{L 1}(t) \geq A_{21}-\left(\beta_{21} \delta+d_{21}\right) S_{L 1}, \\
& \dot{S}_{L 2}(t) \geq A_{22}-\left(\beta_{22} \delta+d_{22}\right) S_{L 2}
\end{aligned}
$$

for $t \geq T$. Consider the following auxiliary system:

$$
\begin{aligned}
\dot{\tilde{S}}_{D 1}(t)= & A_{11}-\left(\beta_{11} \delta+d_{11}\right) \widetilde{S}_{D 1}-D_{1} \widetilde{S}_{D 1}+D_{2} \widetilde{S}_{D 2}, \\
\dot{\widetilde{S}}_{D 2}(t)= & A_{12}-\left(\beta_{12} \delta+d_{12}\right) \widetilde{S}_{D 2}-D_{2} \widetilde{S}_{D 2}+D_{1} \widetilde{S}_{D 1}, \\
& \dot{\widetilde{S}}_{L 1}(t)=A_{21}-\left(\beta_{21} \delta+d_{21}\right) \widetilde{S}_{L 1}, \\
& \dot{\widetilde{S}}_{L 2}(t)=A_{22}-\left(\beta_{22} \delta+d_{22}\right) \widetilde{S}_{L 2} .
\end{aligned}
$$

As in our analysis in Lemma 2, system (51) has a unique positive equilibrium $\left(g_{1}(\delta), g_{2}(\delta), g_{3}(\delta), g_{4}(\delta)\right)$ which is globally stable. By (48) and comparison principle, there is a $\tau>0$ such that $S_{D 1}(t) \geq N_{D 1}^{0}-\theta, S_{D 2}(t) \geq N_{D 2}^{0}-\theta, S_{L 1}(t) \geq S_{L 1}^{0}-\theta$, and $S_{L 2}(t) \geq S_{L 2}^{0}-\theta$ for all $t \geq T+\tau$. Consequently, for $t \geq T+\tau$, we have

$$
\begin{gathered}
\dot{I}_{D 1}(t) \geq \beta_{11}\left(N_{D 1}^{0}-\theta\right) I_{L 1}-\left(d_{11}+\sigma_{1}+D_{1}\right) I_{D 1}+D_{2} I_{D 2} \\
\dot{I}_{L 1}(t) \geq \beta_{21}\left(S_{L 1}^{0}-\theta\right) x_{1}-d_{21} I_{L 1}
\end{gathered}
$$




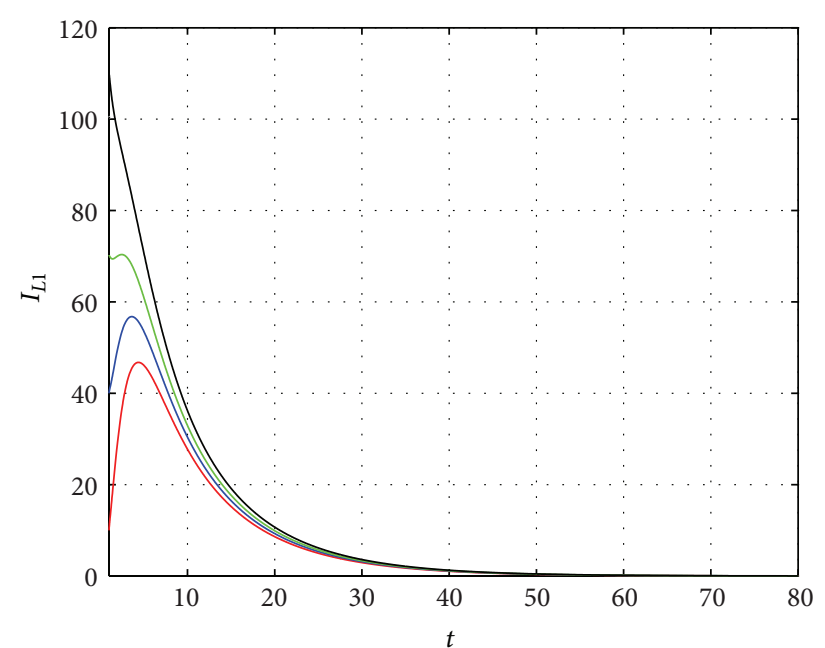

(a)

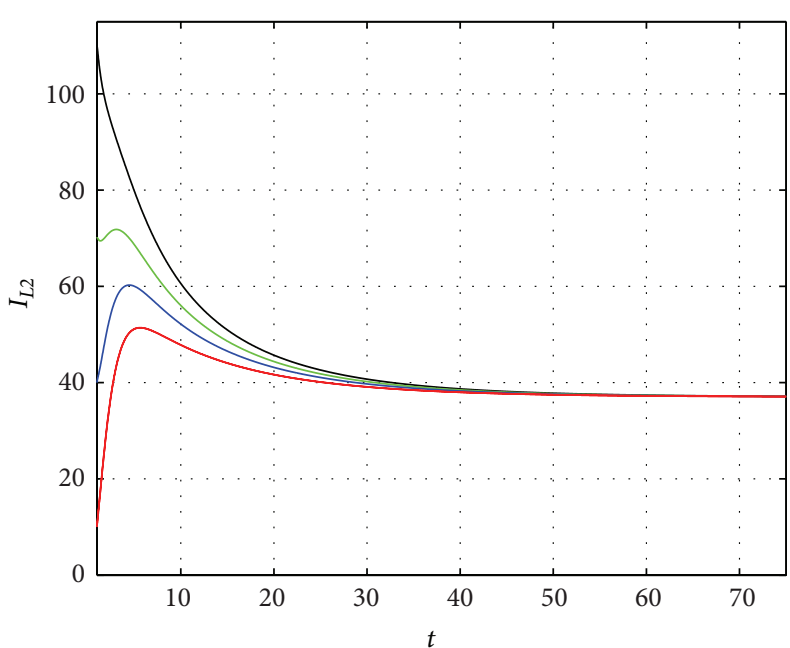

(b)

Figure 2: Time series of echinococcosis disease $I_{L i}, i=1,2$, when the two patches are isolated for the parameters given in Example 8 .

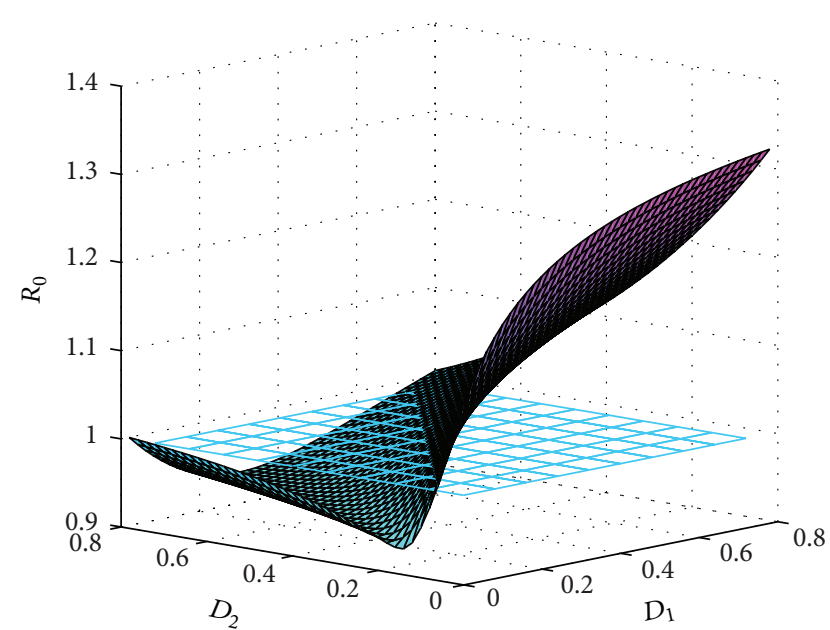

FIgURE 3: Surface plot of $R_{0}$ as a function of $D_{1}$ and $D_{2}$ for the parameters given in Example 8.

$$
\begin{gathered}
\dot{x}_{1}(t) \geq a_{1} I_{D 1}-d_{1} x_{1}, \\
\dot{I}_{D 2}(t) \geq \beta_{12}\left(N_{D 2}^{0}-\theta\right) I_{L 2}-\left(d_{12}+\sigma_{2}+D_{2}\right) I_{D 2}+D_{1} I_{D 1}, \\
\dot{I}_{L 2}(t) \geq \beta_{22}\left(S_{L 2}^{0}-\theta\right) x_{2}-d_{22} I_{L 2}, \\
\dot{x}_{2}(t) \geq a_{2} I_{D 2}-d_{2} x_{2} .
\end{gathered}
$$

Consider an auxiliary system

$$
\begin{gathered}
\dot{\tilde{I}}_{D 1}(t)=\beta_{11}\left(N_{D 1}^{0}-\theta\right) \widetilde{I}_{L 1}-\left(d_{11}+\sigma_{1}+D_{1}\right) \widetilde{I}_{D 1}+D_{2} \widetilde{I}_{D 2} \\
\dot{\tilde{I}}_{L 1}(t)=\beta_{21}\left(S_{L 1}^{0}-\theta\right) \widetilde{x}_{1}-d_{21} \widetilde{I}_{L 1}
\end{gathered}
$$

$$
\begin{gathered}
\dot{\tilde{x}}_{1}(t)=a_{1} \widetilde{I}_{D 1}-d_{1} \widetilde{x}_{1}, \\
\dot{\tilde{I}}_{D 2}(t)=\beta_{12}\left(N_{D 2}^{0}-\theta\right) \widetilde{I}_{L 2}-\left(d_{12}+\sigma_{2}+D_{2}\right) \widetilde{I}_{D 2}+D_{1} \widetilde{I}_{D 1}, \\
\dot{\widetilde{I}}_{L 2}(t)=\beta_{22}\left(S_{L 2}^{0}-\theta\right) \widetilde{x}_{2}-d_{22} \widetilde{I}_{L 2}, \\
\dot{\tilde{x}}_{2}(t)=a_{2} \widetilde{I}_{D 2}-d_{2} \tilde{x}_{2} .
\end{gathered}
$$

The coefficient matrix of the right hand of (53) is $J_{\theta}$. Since matrix $J_{\theta}$ has a positive eigenvalues $s\left(J_{\theta}\right)$ with a positive eigenvector, it follows from a comparison principle that $I_{D i}(t) \rightarrow \infty, I_{L i}(t) \rightarrow \infty$, and $x_{i}(t) \rightarrow \infty$ as $t \rightarrow \infty, i=$ 1,2 , which leads to a contradiction. This proves (49). Hence $W^{s}\left(E_{0}\right) \cap X_{0}=\emptyset$. Clearly, every forward orbit in $M_{\partial}$ converges to $E_{0}$. By Theorem 4.6 in [32] we are able to conclude that model (2) is uniformly persistent with respect to $\left(X_{0}, \partial X_{0}\right)$. Thus, by a well-known result in persistence theory in [33] we know that model (2) has at least one positive equilibrium $E^{*}\left(S_{D 1}^{*}, I_{D 1}^{*}, S_{L 1}^{*}, I_{L 1}^{*}, x_{1}^{*}, S_{D 2}^{*}, I_{D 2}^{*}, S_{L 2}^{*}, I_{L 2}^{*}, x_{2}^{*}\right)$. This completes the proof of Theorem 6 .

\section{Global Stability of $E^{*}$}

We further have the following result on the stability of the endemic equilibrium.

Theorem 7. If $R_{0}>1$, then model (2) admits a unique equilibrium $E^{*}\left(S_{D 1}^{*}, I_{D 1}^{*}, S_{L 1}^{*}, I_{L 1}^{*}, x_{1}^{*}, S_{D 2}^{*}, I_{D 2}^{*}, S_{L 2}^{*}, I_{L 2}^{*}, x_{2}^{*}\right)$, which is globally asymptotically stable.

Proof. In Lemma 3, we have proved that $S_{D i}(t)+I_{D i}(t) \rightarrow$ $N_{D i}^{0}$ and $S_{L i}(t)+I_{L i}(t) \rightarrow S_{L i}^{0}$ as $t \rightarrow \infty, i=1,2$. Therefore, in model (2) we can represent $S_{D i}$ and $S_{L i}$ by $N_{D i}^{0}-I_{D i}(t)$ and 


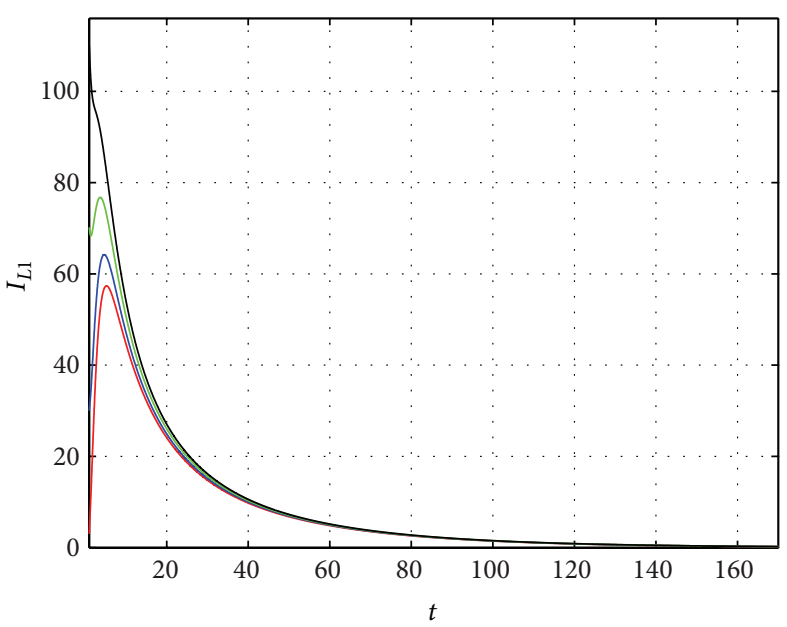

(a)

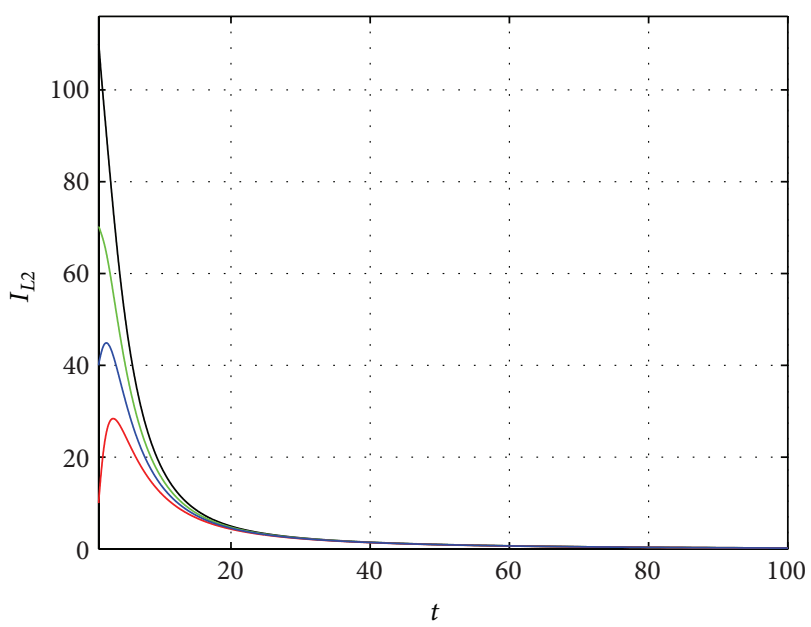

(b)

Figure 4: Time series of echinococcosis disease $I_{L i}, i=1,2$, when the two patches are connected with $D_{1}=0.8, D_{2}=0.2$.

$S_{L i}^{0}-S_{L i}(t), i=1,2$, respectively, and the model (2) will degenerate into the following system with six equations:

$$
\begin{gathered}
\dot{I}_{D 1}(t)=-\left(d_{11}+\sigma_{1}+D_{1}\right) I_{D 1}+\beta_{11}\left(N_{D 1}^{0}-I_{D 1}\right) I_{L 1} \\
+D_{2} I_{D 2} \\
\dot{I}_{L 1}(t)=-d_{21} I_{L 1}+\beta_{21}\left(S_{L 1}^{0}-I_{L 1}\right) x_{1} \\
\dot{x}_{1}(t)=a_{1} I_{D 1}-d_{1} x_{1} \\
\dot{I}_{D 2}(t)=D_{1} I_{D 1}-\left(d_{12}+\sigma_{2}+D_{2}\right) I_{D 2} \\
\dot{I}_{D 1}(t)=+\beta_{12}\left(N_{D 2}^{0}-I_{D 2}\right) I_{L 2} \\
\dot{I}_{L 2}(t)=-d_{22} I_{L 2}+\beta_{22}\left(S_{L 2}^{0}-I_{L 2}\right) x_{2} \\
\dot{x}_{2}(t)=a_{2} I_{D 2}-d_{2} x_{2} .
\end{gathered}
$$

By Lemma 3, the dynamics of system (54) can be focused on the following region:

$$
\begin{gathered}
\Omega=\left\{\left(I_{D 1}, I_{L 1}, x_{1}, I_{D 2}, I_{L 2}, x_{2}\right): 0 \leq I_{D i} \leq N_{D i}^{0},\right. \\
\left.0 \leq I_{L i} \leq S_{L i}^{0}, 0 \leq x_{i} \leq \frac{a_{i}}{d_{i}} N_{D i}^{0}, i=1,2\right\} .
\end{gathered}
$$

We will use the theory of cooperate system to prove the global stability of system (54). Therefore, we only verify the assumption in Corollary 3.2 [34] for system (54). Let

$$
f(u)=\left(\begin{array}{l}
f_{1}\left(u_{1}, u_{2}, u_{3}, u_{4}, u_{5}, u_{6}\right) \\
f_{2}\left(u_{1}, u_{2}, u_{3}, u_{4}, u_{5}, u_{6}\right) \\
f_{3}\left(u_{1}, u_{2}, u_{3}, u_{4}, u_{5}, u_{6}\right) \\
f_{4}\left(u_{1}, u_{2}, u_{3}, u_{4}, u_{5}, u_{6}\right) \\
f_{5}\left(u_{1}, u_{2}, u_{3}, u_{4}, u_{5}, u_{6}\right) \\
f_{6}\left(u_{1}, u_{2}, u_{3}, u_{4}, u_{5}, u_{6}\right)
\end{array}\right)
$$

$$
=\left(\begin{array}{c}
-\left(d_{11}+\sigma_{1}+D_{1}\right) u_{1}+\beta_{11}\left(N_{D 1}^{0}-u_{1}\right) u_{2}+D_{2} u_{4} \\
-d_{21} u_{2}+\beta_{21}\left(S_{L 1}^{0}-u_{2}\right) u_{3} \\
a_{1} u_{1}-d_{1} u_{3} \\
D_{1} u_{1}-\left(d_{12}+\sigma_{2}+D_{2}\right) u_{4}+\beta_{12}\left(N_{D 2}^{0}-u_{4}\right) u_{5} \\
-d_{22} u_{5}+\beta_{22}\left(S_{L 2}^{0}-u_{5}\right) u_{6} \\
a_{2} u_{4}-d_{2} u_{6}
\end{array}\right) \text {, }
$$

and then $f: \mathbb{R}_{+}^{6} \rightarrow \mathbb{R}_{+}^{6}$ is a continuously differentiable map. Clearly $f(0)=0$ and $f_{i}(u) \geq 0$ for all $u \in \Omega$ with $u_{i}=0$, $i=1,2, \ldots, 6$. Since $\partial f_{i} / \partial u_{j} \geq 0(i \neq j)$ for $u \in \Omega$, we have that $f$ is cooperative on $\Omega$. For every $p \in(0,1)$ and $u \in \Omega$, we have

$$
\begin{aligned}
& f_{1}\left(p u_{1}, p u_{2}, p u_{3}, p u_{4}, p u_{5}, p u_{6}\right) \\
& \quad=-\left(d_{11}+\sigma_{1}+D_{1}\right) p u_{1}+\beta_{11}\left(N_{D 1}^{0}-p u_{1}\right) p u_{2}+D_{2} p u_{4} \\
& \quad \geq-\left(d_{11}+\sigma_{1}+D_{1}\right) p u_{1}+\beta_{11}\left(N_{D 1}^{0}-u_{1}\right) p u_{2}+D_{2} p u_{4} \\
& \quad=p f_{1}\left(u_{1}, u_{2}, u_{3}, u_{4}, u_{5}, u_{6}\right) .
\end{aligned}
$$

Using the same argument, we can show that $f$ is strictly sublinear on $\Omega$. By computing $D f(u)$, we have

$$
\left(\frac{\partial f_{i}}{\partial u_{j}}\right)_{1 \leq i, j \leq 6}=\left(\begin{array}{ll}
f_{11}(u) & f_{12}(u) \\
f_{21}(u) & f_{22}(u)
\end{array}\right),
$$




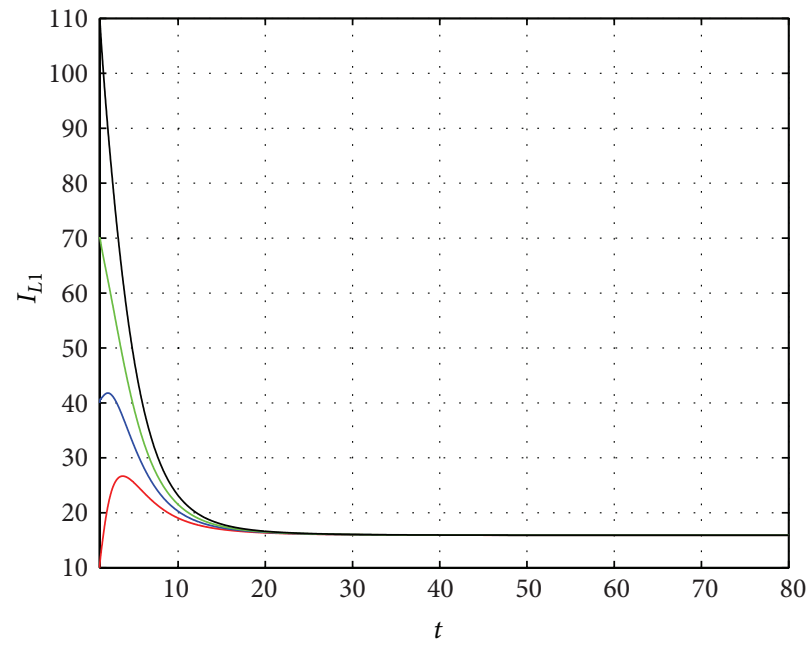

(a)

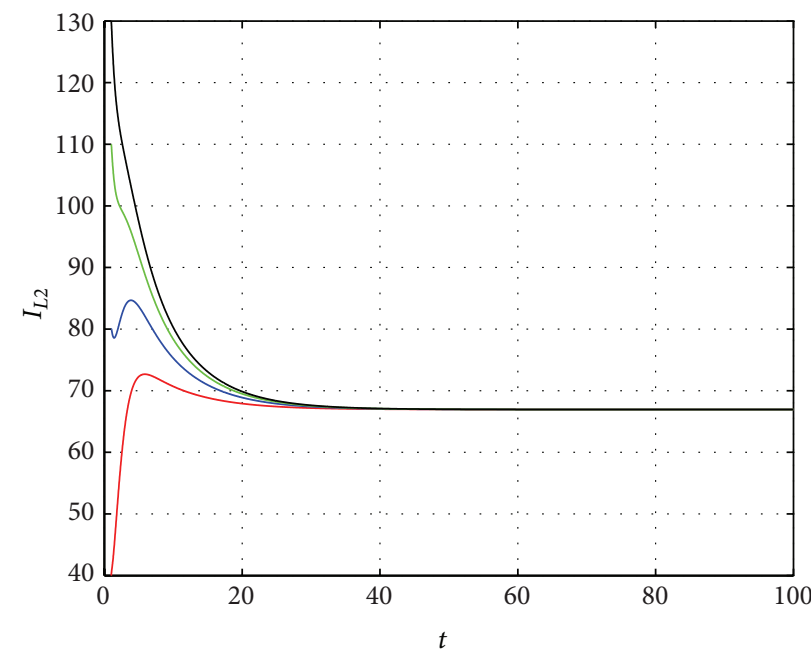

(b)

Figure 5: Time series of echinococcosis disease $I_{L i}, i=1,2$, when the two patches are connected with $D_{1}=0.2, D_{2}=0.8$.

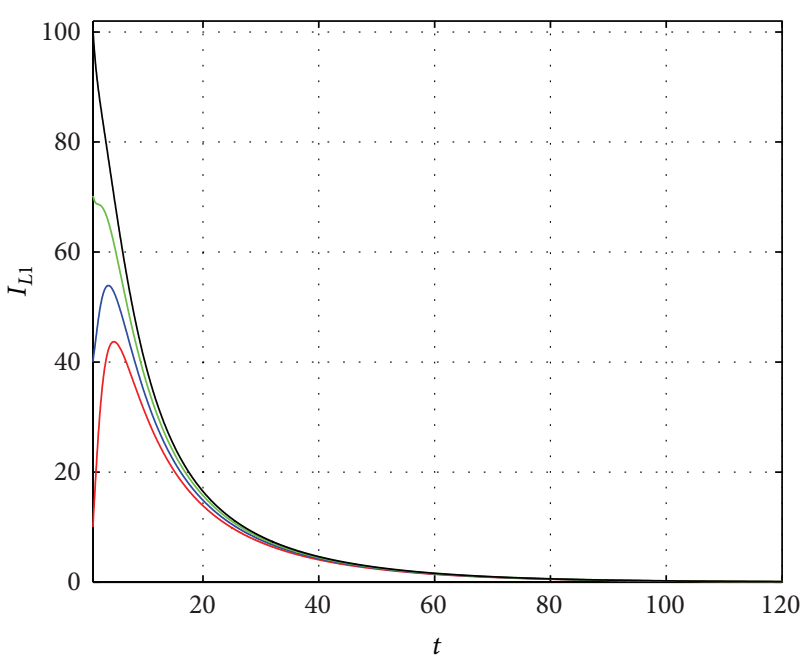

(a)

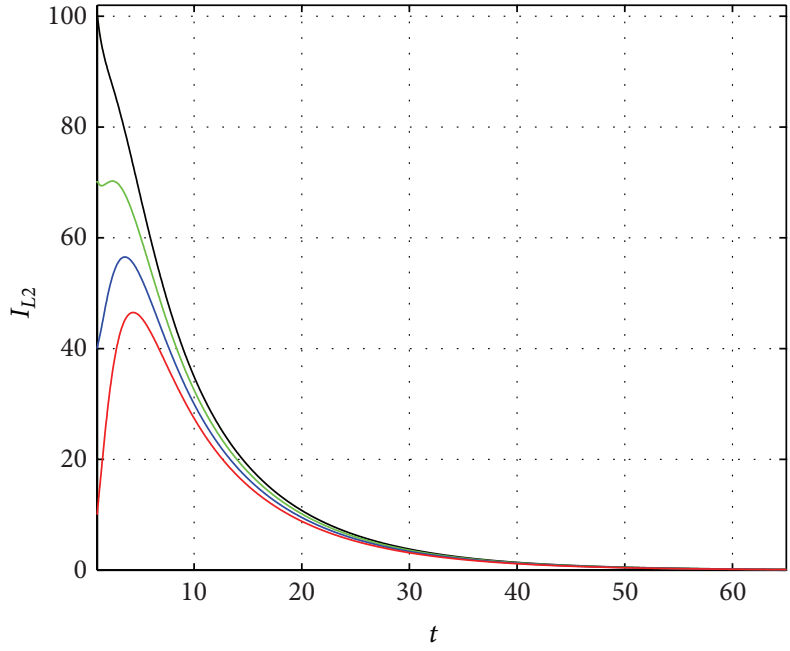

(b)

FIgURE 6: Time series of echinococcosis disease $I_{L i}, i=1,2$, when the two patches are isolated for the parameters given in Example 9 .

where

$$
\begin{aligned}
& f_{11}(u)=\left(\begin{array}{ccc}
-\left(d_{11}+\sigma_{1}+D_{1}\right)-\beta_{11} u_{2} & \beta_{11}\left(N_{D 1}^{0}-u_{1}\right) & 0 \\
0 & -d_{21}-\beta_{21} u_{3} & \beta_{21}\left(S_{L 1}^{0}-u_{3}\right) \\
a_{1} & 0 & -d_{1}
\end{array}\right), \\
& f_{12}(u)=\left(\begin{array}{ccc}
D_{2} & 0 & 0 \\
0 & 0 & 0 \\
0 & 0 & 0
\end{array}\right), \quad f_{21}(u)=\left(\begin{array}{ccc}
D_{1} & 0 & 0 \\
0 & 0 & 0 \\
0 & 0 & 0
\end{array}\right) \\
& f_{22}(u)=\left(\begin{array}{ccc}
-\left(d_{12}+\sigma_{2}+D_{2}\right)-\beta_{12} u_{5} & \beta_{12}\left(N_{D 2}^{0}-u_{4}\right) & 0 \\
0 & -d_{22}-\beta_{22} u_{6} & \beta_{22}\left(S_{L 2}^{0}-u_{5}\right) \\
a_{2} & 0 & -d_{2}
\end{array}\right) .
\end{aligned}
$$




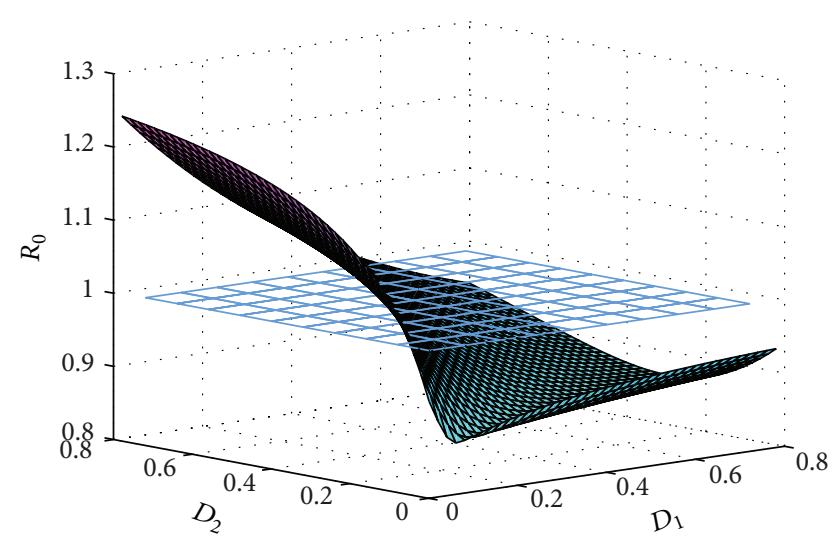

Figure 7: Surface plot of $R_{0}$ as a function of $D_{1}$ and $D_{2}$ for the parameters given in Example 9.

Clearly, $D f(u)$ is irreducible for $u \in \Omega$. From (24) we can see that if $R_{0}>1$, then $s(J)>0$. Since $D f(0)=J$, we have $s(D f(0))=s(J)>0$. By Corollary 3.2 in [34], one can conclude that system (54) admits a unique positive equilibrium $\left(I_{D 1}^{*}, I_{L 1}^{*}, x_{1}^{*}, I_{D 2}^{*}, I_{L 2}^{*}, x_{2}^{*}\right)$, which is globally asymptotically stable. According to the theory of asymptotic autonomous systems [31], we further obtain that endemic equilibrium $E^{*}\left(S_{D 1}^{*}, I_{D 1}^{*}, S_{L 1}^{*}, I_{L 1}^{*}, x_{1}^{*}, S_{D 2}^{*}, I_{D 2}^{*}, S_{L 2}^{*}, I_{L 2}^{*}, x_{2}^{*}\right)$ is globally attractive for model (2).

\section{Simulations}

To complement the mathematical analysis carried out in the previous section, we now investigate some of the numerical properties of the two-patch model (2).

Example 8. Take parameters in model (2) as follows:

$A_{11}=15, \beta_{11}=0.00065, d_{11}=0.3, \sigma_{1}=0.2, A_{21}=80$, $\beta_{21}=0.004, d_{21}=0.4, a_{1}=150, d_{1}=33, A_{12}=15, \beta_{12}=$ $0.0015, d_{12}=0.3, \sigma_{2}=0.2, A_{22}=80, \beta_{22}=0.004, d_{22}=0.4$, $a_{2}=150$, and $d_{2}=33$. If the two patches are isolated, by simple calculations we have $R_{01}=0.8392, R_{02}=1.1089$.

From Lemma 1 we have that the disease will die out in the first patch and will be endemic in the second patch (see Figure 2). From Figure 3 we can easily see that $R_{0}$ will be larger than 1 under the condition of a larger $D_{1}$ and a smaller $D_{2}$. This means that the larger diffusion of dogs from the lower epidemic areas to the higher prevalence areas can intensify the spread of echinococcosis (see Figure 4). However, when $D_{1}$ is small and $D_{2}$ is large, $R_{0}$ will be smaller than 1 . This indicates that the larger diffusion of dogs from the higher prevalence areas to the lower epidemic areas can reduce the spread and is beneficial for disease control (see Figure 5).

Example 9. We use the parameters given in Example 8 except that $A_{11}=10, \beta_{11}=0.0015, A_{21}=70, A_{12}=20$, and $\beta_{12}=$ 0.0005 . If the two patches are isolated, by simple calculations we have $R_{01}=0.9266, R_{02}=0.8463$.

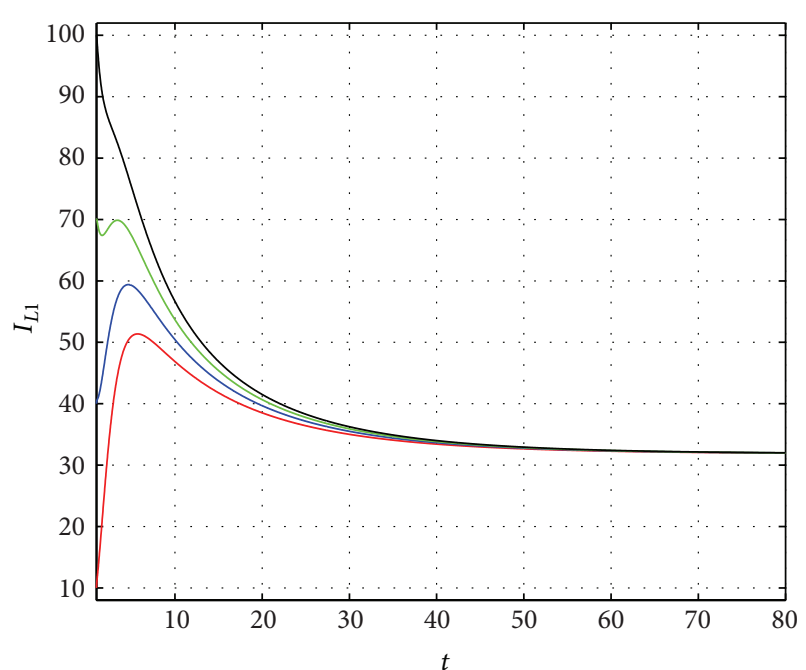

(a)

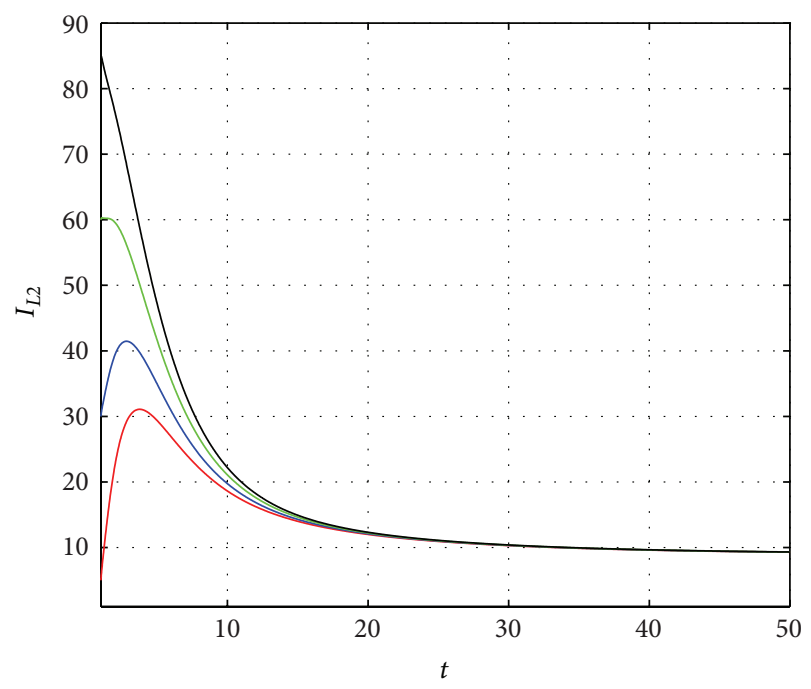

(b)

Figure 8: Time series of echinococcosis disease $I_{L i}, i=1,2$, when the two patches are connected with $D_{1}=0.2, D_{2}=0.6$.

It follows from Lemma 1 that the disease will die out in both two patches when they are isolated (see Figure 6). However, from Figure 7 we can see that $R_{0}$ is not always less than 1 . This suggests that dogs diffusion can cause the spread of echinococcosis in two patches (see Figure 8).

\section{Discussion}

In this paper, in order to model the transmission dynamics of echinococcosis spread between two patches due to dogs migration a patch model for echinococcosis is proposed. We define the basic reproduction number $R_{0}$. The mathematical results show that the dynamics of the model is completely determined by $R_{0}$. If $R_{0}<1$, the disease-free equilibrium is globally asymptotically stable. When $R_{0}>$ 1 , the model is permanence and endemic equilibrium is globally asymptotically stable. According to the simulation 
we have that the larger diffusion of dogs from a low epidemic area to the high prevalence area can intensify the disease spread. However, the larger diffusion of dogs from the high prevalence area to a low epidemic area can reduce the disease spread and is beneficial to disease control. Additionally, the model presented in this paper can be extended to describe the dynamical transmission of echinococcosis with dogs migration among more than two patches. We leave these in our future work.

\section{Conflict of Interests}

The authors declare that they have no financial and personal relationships with other people or organizations that can inappropriately influence their work and there are no professional or other personal interests of any nature or kind in any product, service, and/or company that could be construed as influencing the position presented in, or the review of, this paper.

\section{Acknowledgments}

This work is State Key Laboratory Basic Medicine Base of Xinjiang Hydatid Disease Research Fund (XJDX02022013-2), Cultivation Project Young Scientific Innovative Talents (2013731017), the National Natural Science Foundation of China (11201399, 11271312), the Scientific Research Programmes of Colleges in Xinjiang (XJEDU2012S20), and Academic Discipline Project of Xinjiang Medical University Health Measurements and Health Economics (XYDXK50780308).

\section{References}

[1] S. A. Berger and J. S. Marr, Human Parasitic Diseases Sourcebook, Jones \& Bartlett Learning, Sudbury, Mass, USA, 2006.

[2] C. N. Macpherson, "An active intermediate host role for man in the life cycle of Echinococcus granulosus in Turkana, Kenya," American Journal of Tropical Medicine and Hygiene, vol. 32, no. 2, pp. 397-404, 1983.

[3] W. Zhenghuan, W. Xiaoming, and L. Xiaoqing, "Echinococcosis in China, a review of the epidemiology of Echinococcus spp.", EcoHealth, vol. 5, no. 2, pp. 115-126, 2008.

[4] M. Roberts, J. Lawson, and M. Gemmell, "Population dynamics in Echinococcosis and cysticercosis: mathematical model of the life-cycle of Echinococcus granulosus," Parasitology, vol. 92, no. 3, pp. 621-641, 1986.

[5] P. Torgerson, B. Shaikenov, A. Rysmukhambetova, A. Ussenbayev, A. M. Abdybekova, and K. K. Burtisurnov, "Modelling the transmission dynamics of Echinococcus granulosus in dogs in rural Kazakhstan," Parasitology, vol. 126, no. 5, pp. 417-424, 2003.

[6] P. Torgerson, "Canid immunity to Echinococcus spp.: impact on transmission," Parasite Immunology, vol. 28, no. 7, pp. 295-303, 2006.

[7] P. Torgerson, "The use of mathematical models to simulate control options for echinococcosis," Acta Tropica, vol. 85, no. 2, pp. 211-221, 2003.

[8] D. Heinzmann, A. D. Barbour, and P. R. Torgerson, "A mechanistic individual-based two-host interaction model for the transmission of a parasitic disease," International Journal of Biomathematics, vol. 4, no. 4, pp. 443-460, 2011.

[9] M. Roberts and M. Aubert, "A model for the control of echinococcus multilocularis in France," Veterinary Parasitology, vol. 56, no. 1-3, pp. 67-74, 1995.

[10] M. Vervaeke, S. Davis, H. Leirs, and R. Verhagen, "Implications of increased susceptibility to predation for managing the sylvatic cycle of Echinococcus multilocularis," Parasitology, vol. 132, no. 6, pp. 893-901, 2006.

[11] H. Ishikawa, Y. Ohga, and R. Doi, "A model for the transmission of Echinococcus multilocularis in Hokkaido, Japan," Parasitology Research, vol. 91, no. 6, pp. 444-451, 2003.

[12] T. Nishina and H. Ishikawa, "A stochastic model of Echinococcus multilocularis transmission in Hokkaido, Japan, focusing on the infection process," Parasitology Research, vol. 102, no. 3, pp. 465-479, 2008.

[13] F. Hansen, K. Tackmann, F. Jeltsch, C. Wissel, and H. Thulke, "Controlling echinococcus multilocularis-ecological implications of field trials," Preventive Veterinary Medicine, vol. 60, no. 1, pp. 91-105, 2003.

[14] K. Takumi and J. Van der Giessen, "Transmission dynamics of Echinococcus multilocularis; its reproduction number, persistence in an area of low rodent prevalence, and effectiveness of control," Parasitology, vol. 131, no. 1, pp. 133-140, 2005.

[15] K. Takumi, A. de Vries, M. L. Chu, J. Mulder, P. Teunis, and J. van der Giessen, "Evidence for an increasing presence of Echinococcus multilocularis in foxes in The Netherlands," International Journal for Parasitology, vol. 38, no. 5, pp. 571-578, 2008.

[16] N. Kato, K. Kotani, S. Ueno, and H. Matsuda, "Optimal risk management of human alveolar echinococcosis with vermifuge," Journal of Theoretical Biology, vol. 267, no. 3, pp. 265271, 2010

[17] J. A. M. Atkinson, G. M. Williams, L. Yakob et al., "Synthesising 30 years of mathematical modelling of Echinococcus transmission," PLoS Neglected Tropical Diseases, vol. 7, no. 8, p. e2386, 2013.

[18] K. Wang, X. Zhang, Z. Jin, H. Ma, Z. Teng, and L. Wang, "Modeling and analysis of the transmission of Echinococcosis with application to Xinjiang Uygur Autonomous Region of China," Journal of Theoretical Biology, vol. 333, pp. 78-90, 2013.

[19] W. Wang and X.-Q. Zhao, "An epidemic model in a patchy environment," Mathematical Biosciences, vol. 190, no. 1, pp. 97$112,2004$.

[20] Y. Jin and W. Wang, "The effect of population dispersal on the spread of a disease," Journal of Mathematical Analysis and Applications, vol. 308, no. 1, pp. 343-364, 2005.

[21] J. Cui, Y. Takeuchi, and Y. Saito, "Spreading disease with transport-related infection," Journal of Theoretical Biology, vol. 239, no. 3, pp. 376-390, 2006.

[22] Y. Yang, J. Wu, J. Li, and Z. Ma, "Global dynamics-convergence to equilibria-of epidemic patch models with immigration," Mathematical and Computer Modelling, vol. 51, no. 5-6, pp. 329337,2010

[23] D. Gao and S. Ruan, "An SIS patch model with variable transmission coefficients," Mathematical Biosciences, vol. 232, no. 2, pp. 110-115, 2011.

[24] J. J. Tewa, S. Bowong, and B. Mewoli, "Mathematical analysis of two-patch model for the dynamical transmission of tuberculosis," Applied Mathematical Modelling, vol. 36, no. 6, pp. 24662485, 2012. 
[25] R. Lintott, R. Norman, and A. Hoyle, "The impact of increased dispersal in response to disease control in patchy environments," Journal of Theoretical Biology, vol. 323, pp. 57-68, 2013.

[26] Z. Qiu, "Dynamics of an epidemic model with host migration," Applied Mathematics and Computation, vol. 218, no. 8, pp. 46144625, 2011.

[27] J. Liu, "Spread of disease in a patchy environment," Chaos, Solitons and Fractals, vol. 45, no. 7, pp. 942-949, 2012.

[28] D. Gao and S. Ruan, "A multipatch Malaria model with logistic growth populations," SIAM Journal on Applied Mathematics, vol. 72, no. 3, pp. 819-841, 2012.

[29] O. Diekmann, J. A. P. Heesterbeek, and J. A. J. Metz, "On the definition and the computation of the basic reproduction ratio $R_{0}$ in models for infectious diseases in heterogeneous populations," Journal of Mathematical Biology, vol. 28, no. 4, pp. 365-382, 1990.

[30] P. Van den Driessche and J. Watmough, "Reproduction numbers and sub-threshold endemic equilibria for compartmental models of disease transmission," Mathematical Biosciences, vol. 180, pp. 29-48, 2002.

[31] H. R. Thieme, "Convergence results and a Poincaré-Bendixson trichotomy for asymptotically autonomous differential equations," Journal of Mathematical Biology, vol. 30, no. 7, pp. 755763, 1992.

[32] H. R. Thieme, "Persistence under relaxed point-dissipativity (with application to an endemic model)," SIAM Journal on Mathematical Analysis, vol. 24, no. 2, pp. 407-435, 1993.

[33] V. Hutson and K. Schmitt, "Permanence and the dynamics of biological systems," Mathematical Biosciences, vol. 111, no. 1, pp. 1-71, 1992.

[34] X. Q. Zhao and Z. J. Jing, "Global asymptotic behavior in some cooperative systems of functional-differential equations," The Canadian Applied Mathematics Quarterly, vol. 4, no. 4, pp. 421444, 1996. 


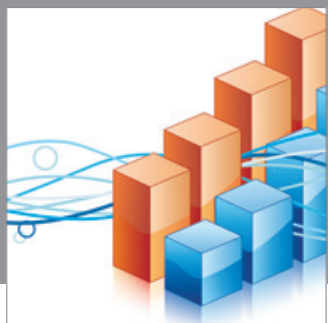

Advances in

Operations Research

mansans

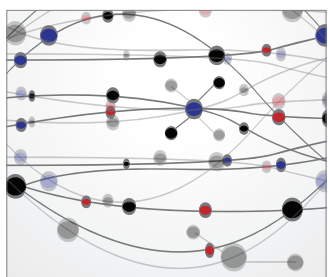

The Scientific World Journal
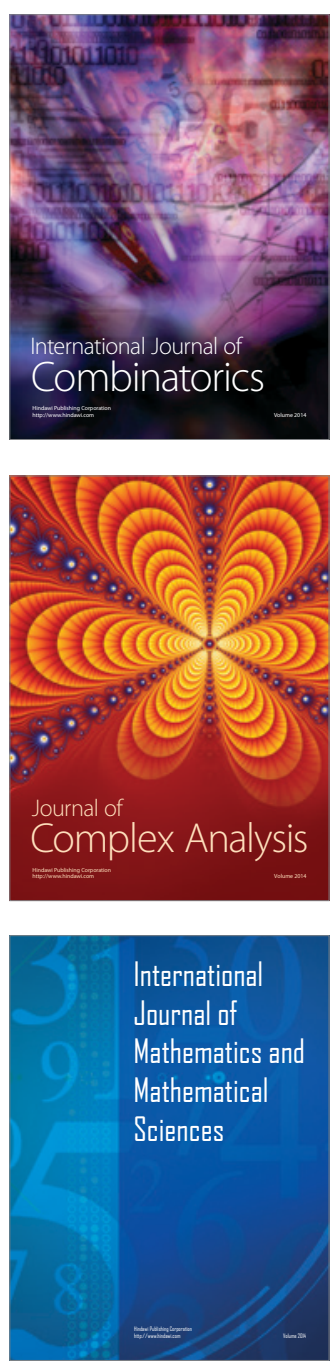
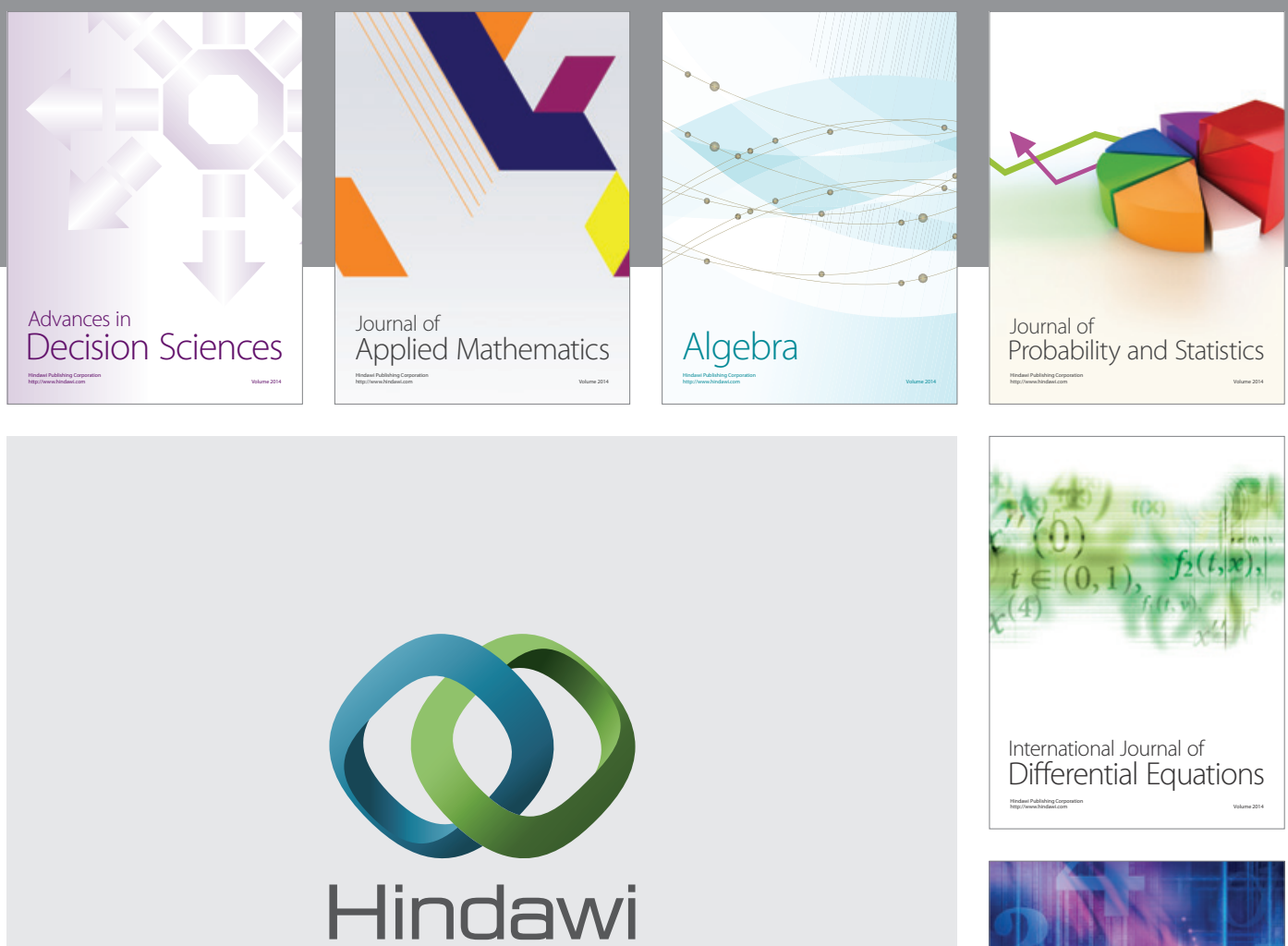

Submit your manuscripts at http://www.hindawi.com
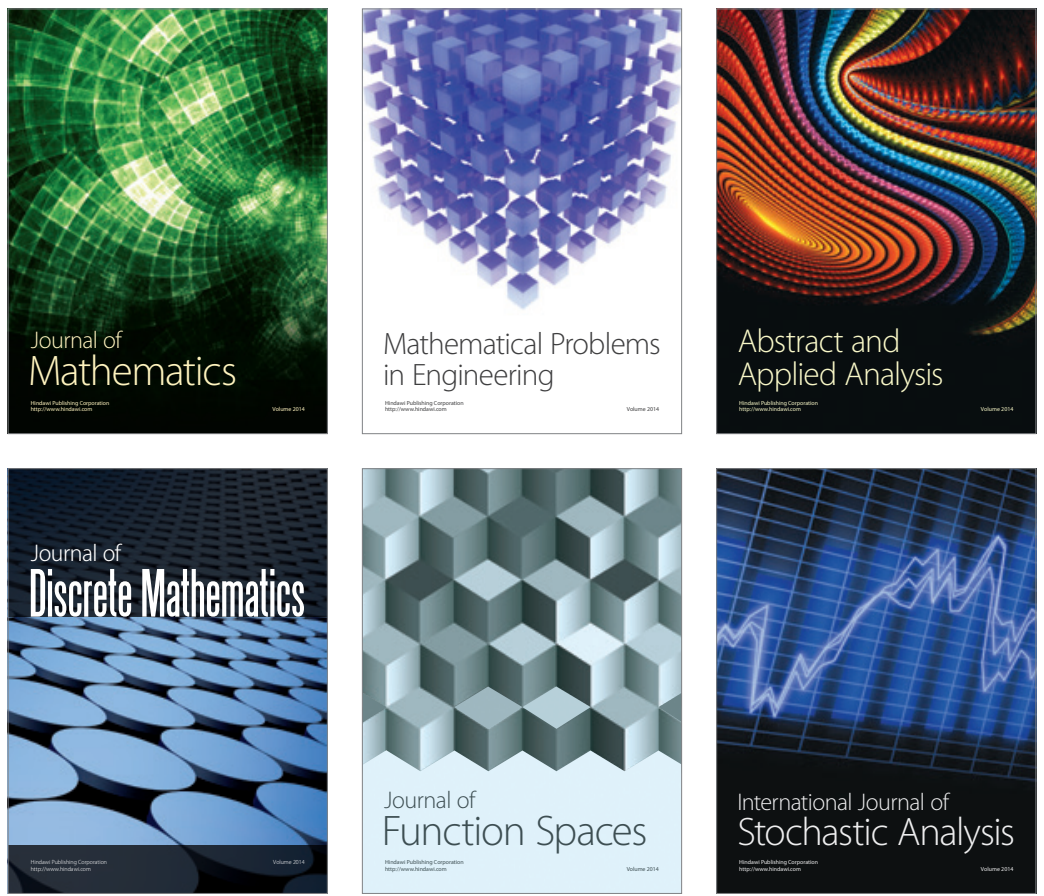

Journal of

Function Spaces

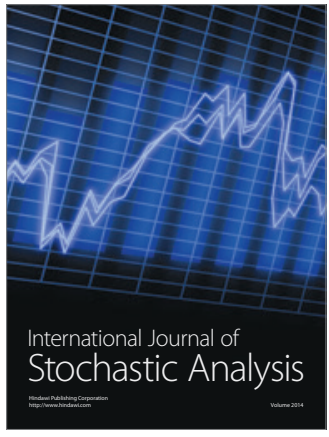

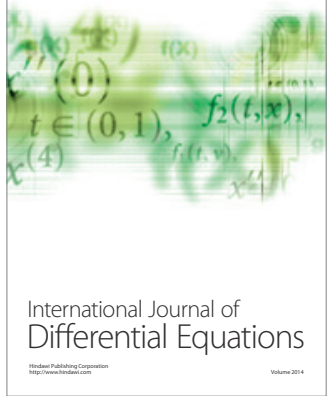
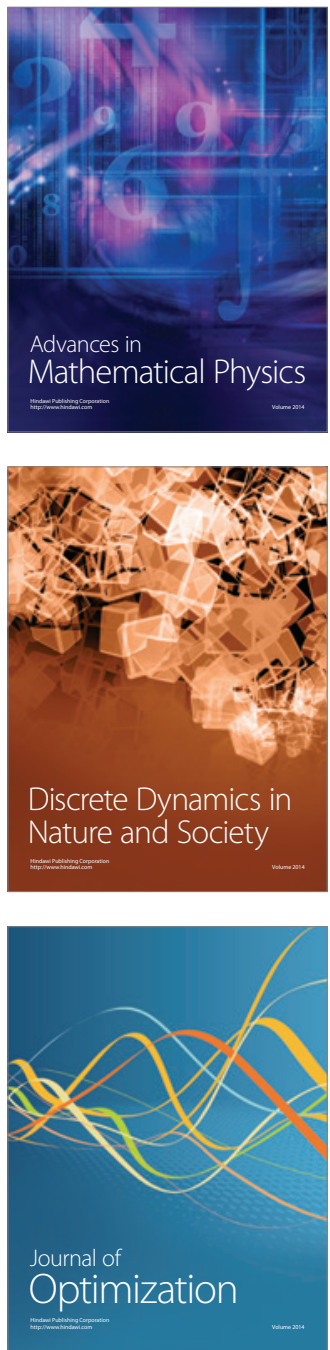